\title{
$\Omega$
}

Georgia Hardavella ${ }^{1,2}$, Ane Aamli-Gaagnat ${ }^{3}$, Neil Saad $^{4}$, Ilona Rousalova ${ }^{5}$, Katherina B. Sreter ${ }^{6}$

(1)

1'Dept of Respiratory Medicine, King's College Hospital, London, UK. ²Dept of Respiratory Medicine and Allergy, King's

College London, London, UK. ${ }^{3}$ Dept of Clinical Science, University of Bergen, Bergen, Norway. ${ }^{4}$ Faculty of Medicine,

National Heart and Lung Institute, Imperial College, London, UK. ${ }^{5} 1$ st Dept of Tuberculosis and Respiratory Care,

1st Medical School and General University Hospital, Charles University in Prague, Prague, Czech Republic. ${ }^{6}$ Dept of Clinical

Immunology, Pulmonology and Rheumatology, University Hospital Centre "Sestre Milosrdnice”, Zagreb, Croatia.

\section{How to give and receive feedback effectively}

\section{Doing science}

Feedback is the breakfast of champions.

Ken Blanchard

Feedback is the fuel that drives improved performance.

Eric Parsloe

In most European countries, feedback is embedded in education, training and daily professional activities. It is a valuable tool for indicating whether things are going in the right direction or whether redirection is required. In the world of healthcare professionals, it is intended to provide doctors with information about their practice through the eyes of their peers. Feedback is a valuable tool for doctors to gather information, consolidate their awareness of strengths and areas to improve, and aims to support effective behaviour. Doctors of all levels may be approached by peers or juniors to give feedback, or they may ask others to give feedback on their own performance.

Giving and receiving feedback is not an easy task and poses significant challenges for both sides. In this article, we will discuss pragmatic feedback models, how to overcome barriers to an effective feedback and tips for giving effective feedback, as well as how to receive feedback and make the most out of it.

\section{Types of feedback}

- Informal feedback is the most frequent form. It is provided on a day-to-day basis, and is given on any aspect of a doctor's professional performance and conduct, by any member of the multidisciplinary team. It is usually in verbal form.

- Formal feedback comes as part of a structured assessment; it can be offered by any member of the multidisciplinary team, but most frequently by peers or superiors. It is usually in written form.

- Formative feedback, "for learning", is about a learner's progress at a particular time through a course or during the acquisition of a new skill. It provides opportunities to gain feedback, reflect and redirect effort (where appropriate) before completing a final assessment. It gives you the experience of writing or performing a task without it having a direct impact on your formal progress and relies on continuous encouragement.

- Summative feedback, "of learning", measures performance, often against a standard, and comes with a mark/grade and feedback to explain your mark. It can be used to rank or judge individuals

For the purpose of this article, we will focus on formal and informal feedback.

\section{Why is feedback important?}

As a general rule, it seems that learners value feedback more when it is given by someone they respect as a role model. Appropriate feedback contributes significantly in developing learners' competence and confidence at all stages of their professional careers; it helps them think about the gap between
Cite as: Hardavella $G$, Aamli-Gaagnat A, Saad N, et al. How to give and receive feedback effectively. Breathe 2017; 13: 327-333. 
actual and desired performance, and identify ways to narrow the gap and improve. For health professionals in particular, it promotes reflective and experiential learning ,which involves "training on the job", and reflecting on experiences, incidents and feelings. More importantly, feedback aims to develop performance to a higher level by dealing with underperformance in a constructive way.

If we do not give feedback, this will come with a cost. The learner can assume that everything is fine and will continue practicing in the same way. This leads into a false assessment of their own skills and abilities, and builds up a false perception.

\section{Who gives feedback?}

In professional life, your patients and anyone working with you as a member of the multidisciplinary team can give you feedback. Feedback in these cases is meant to be given reciprocally, i.e. you will also need to give feedback to your peers. We have listed the most common sources of feedback in figure 1 .

\section{Educational or clinical supervisors}

Your educational or clinical supervisor can be a major source of feedback. They are meant to act as your mentor, monitor your clinical and educational progress, and ensure you receive appropriate career guidance and planning. Educational or clinical supervisors do not formally exist in all respiratory medicine training programmes across Europe and this role may be informally undertaken ad hoc by the clinical lead of the department, a supervising consultant or a PhD/MSc supervisor. Regardless of the role allocation, feedback is integral to the process, and should cover clinical and academic practice, professional conduct, complaints and/or serious incidents that should be discussed in a reflective, nonjudgmental manner to allow improvement and personal development. Should this occur in a structured and organised manner with a delegated supervisor, it will be helpful for the learner.

\section{Trainees}

As previously stated, the feedback process should be reciprocal. Departmental trainee feedback is

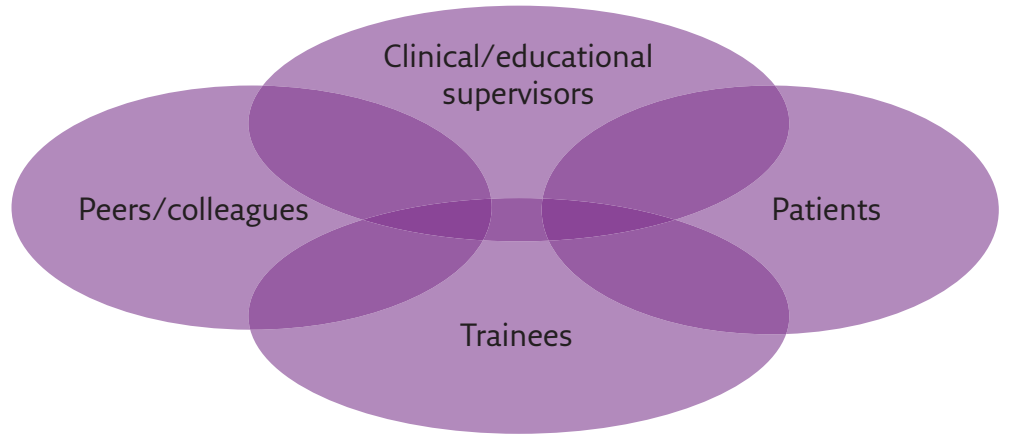

Figure 1 Sources offeedback. essential to monitor and improve the quality of specialty training. Trainees' feedback must be used with other sources of information to review and improve the training programmes and posts. Obtaining this type of feedback can sometimes be challenging as it may be biased by the fear of identification and labelling of trainees; therefore, in some countries, it is given anonymously. This feedback should also be an opportunity to raise concerns about patient safety or colleague bullying and undermining.

\section{Peers and colleagues}

People you work with as members of a multidisciplinary team are expected to provide their feedback in a constructive manner, being open and supportive. This multisource feedback aims to improve your own understanding of where things stand; it is a clear "reality check" and, at the same time, gives a clear direction of travel in terms of improving behaviours, attitudes and skills. If you have not received such feedback, ask for it.

\section{Patients}

Patients' feedback provides valuable information about what patients and service users think about the healthcare services offered. Examining patients' feedback will give a direct insight into what is working well and what needs further improvement in the way care is delivered. Furthermore, patient feedback to healthcare professionals is also important as it highlights examples of good practice where lessons can be learnt and areas of concern where improvements can be made. Measuring patients' feedback and experiences of care/treatment highlights areas that need to improve to provide a patient-led healthcare service.

\section{Feedback models}

There are several different models of giving feedback. Not every model is applicable in all daily cases of providing feedback. Below we have listed some feedback models.

\section{The "feedback sandwich"}

The feedback sandwich starts and concludes with positive feedback, and what can be considered as the more critical feedback is "sandwiched" between the positive aspects. This can be applicable in everyday clinical practise. However, if you use this method continuously, it might lose its effectiveness. The person receiving our feedback will only wait for the "but" in the middle of your sentence. In this case, make sure to give positive feedback on its own when the opportunity arises. If your coworkers feel acknowledged daily, they will be more open to all kinds of feedback when 
applicable. A couple of examples of the feedback sandwich follow.

"You have done really well in the acute take; you prioritised cases efficiently and your management plans were well structured. I was thinking we could discuss a few things I believe you can further improve such as timely communication of messages to the nursing staff and emphasising the urgency of particular tasks. After completing your management plan in the medical notes, you can consider discussing verbally the urgency of some tasks with the nurse looking after the patient so that they are alerted and can proceed with them promptly rather than relying on them going back to read the medical notes, which can take longer due to the overwhelming emergency department. Additional verbal communication will complement the excellent quality of your work, will ensure your well-structured management plan is implemented in a timely manner for the patient's benefit and will further improve your patient's outcome."

"I noticed you made the patient and relatives feel very comfortable while you explained the bronchoscopy test to them, and your explanation was very clear. It would have helped further if you had given them patient information leaflets, as at times they were looking a bit overwhelmed. However, you have set a time for meeting with them again, and this will give you the opportunity of answering any questions and giving the leaflets."

\section{“Chronological fashion" feedback}

Chronological fashion feedback focuses on reflecting observations chronologically, reiterating the events that occurred during the session back to the learner. For instance, an observer can go through a learning session and give feedback from beginning to the end.

"The first thing you did really well when you entered the room was to introduce yourself to the patient. Then, you proceeded with physical examination without getting the patient's consent for this and at that point, the patient looked distressed. It was after that when you explained what you would do and got their consent, and consequently, the patient was more relaxed during the rest of the physical examination."

This is helpful for short feedback sessions but you can become bogged down in detail during long sessions.

\section{Pendleton model}

The Pendleton model was developed in 1984. It is more learner centred, conversation based and identifies an action plan or goals: "reflection for action". The facilitator needs to check whether the learner wants and is ready for feedback. Then, the learner gives some background about what is being assessed and states what was done well. This aims to create a safe environment first by highlighting positives and consequently this prevents defensiveness. The facilitator then reinforces these positives and the learner suggests what could be improved. This is important, as weaknesses are dissected to offer opportunities for reflection. The facilitator advises how this could be improved and a mutually agreed action plan is formed. The main idea is to use open questions and give the learner the opportunity to think and reflect.

"What do you think went well?"

"What do you think could be done differently?"

"What could be further improved?"

"How can this be achieved?"

\section{Barriers to effective feedback}

Various factors can impact on effective feedback and act as barriers (table 1). It is important to be able to identify and overcome them. Effective feedback is dependent on communication skills and as such, it is vital that the message intended by the sender is understood by the receiver in the same terms.

\section{Generalised feedback that is not related to specific facts and does not give advice on how to improve behaviour}

Generalised feedback is unhelpful and can be confusing. The person receiving feedback remains unclear about the actual purpose of the session and usually starts exploring hidden agendas that might have triggered the feedback. It disrupts professional relationships and causes unnecessary suspicion.

Table 1 Barriers to effective feedback

Generalised feedback not related to specific facts

Lack of advice on how to improve behaviour

A lack of respect for the source of feedback

Fear of upsetting colleagues

Fear of damaging professional relationships

Defensive behaviour/resistance when receiving feedback

Physical barriers: noise, or improper time, place or space

Personal agendas

Lack of confidence 


\section{A lack of respect for the source of feedback}

We all tend to accept feedback more from people we value. In the opposite case, it is advised that you ask another colleague that was present to provide informal feedback rather than doing it yourself as otherwise, this might impact on professional relationships and feedback will be ignored.

\section{Fear of upsetting your colleague or damaging your professional relationship with them}

The person giving feedback might be different from the recipient in terms of sex, age, hierarchy, and educational and cultural background. These factors may result in a demotivating feedback session. Therefore, feedback needs to be given in a supportive, empathic and relaxed manner, and on a background of a working relationship based on mutual respect.

\section{The recipient of feedback being resistant or defensive when receiving it}

Poor handling of situations in which the recipient is resistant or defensive can result in a dismissive approach; therefore, feedback will be disregarded.

\section{Physical barriers}

Giving feedback loudly in a noisy corridor, or in the presence of other colleagues or patients, is inappropriate. Such feedback loses its objectivity and the recipient may consider this as an insult that will impact their professional relationship with their peers and patients.

\section{Language barrier or lack of knowledge regarding cultural diversity}

Language and cultural barriers convey unclear messages and result in unclassified assumptions.

Table 2 Tips on giving effective feedback

Plan in advance
Give promptly, right after the event
Think about what you want to achieve and drive discussion accordingly
One-on-one feedback is preferable
Start gently
Be specific
Encourage self-reflection
Be aware of nonverbal clues
Self-reflect after the feedback session is completed

It is important to confirm the message sent is the message that is actually received. All feedback sessions should be held in a respectful and supportive manner.

\section{Personal agendas}

Personal agendas should not influence feedback. As soon as you realise this is a possibility, it is best not to give feedback as this will be perceived by the recipient negatively. Personal reflection will identify the reasons behind this and will be crucial in improving this aspect.

\section{Lacking confidence}

A person given feedback who lacks confidence may exhibit shyness, difficulty in being assertive, or lack of awareness of their own rights and opportunities.

\section{Tips for and principles of giving effective feedback}

When preparing to give feedback, think about what you would like to achieve. What do you want to highlight, what went well and where could there be some improvements? Table 2 summarises tips on giving feedback. Planning in advance is crucial to the process. Planning should encounter to whom you are giving feedback. There is no "one-size fits all" approach; feedback should be tailored to each individual and the corresponding situation. It is of vital importance that you reflect carefully about how you want to convey your message and focus on a couple of key points without overwhelming the learner. The learner might not be ready (i.e. not receptive), which could have adverse effects. Therefore, think about how they will react to the feedback and what your response might be.

Generally, you will provide one-on-one feedback and you must make sure to give it privately. Offering public feedback will only be perceived as a criticism by the learner and overall it may have detrimental effects on departmental relationships. The learner may feel insulted and undermined whereas their self-confidence will also be affected as they feel this may result in losing their colleagues' respect. On some occasions, it is possible to give group feedback but then you need to restrict your feedback to the group as a whole without singling out individuals.

It is best to ensure that feedback is given in a timely manner, i.e. as soon after the event as possible, and most organisations will have a regular feedback scheduled (weekly or monthly). However, should the situation mandate it, you can schedule an ad hoc session. Feedback and reflection work best when the memory is still fresh. When feedback is given with great delay (i.e. months after the incident), then its objectivity will be debated.

In preparing for the feedback, think of specific situations and, if you want to highlight some 
Table 3 Open-ended questions for giving feedback that encourage self-reflection

How do you think things went?

Did it go as planned? If not, why not?

If you were doing it again what would you do the same next time and what would you do differently? Why?

How did you feel during the session? How would you feel about doing it again?

How do you think the patient felt? What makes you think that?

What did you learn from this session?

negative actions, potential alternatives. Do not bring up past actions unless you wish to underscore a certain behaviour or pattern. Also, focus on tasks, actions and objective events rather than personality traits, which tend to be more subjective.

When giving feedback, start off gently trying to implement one of the feedback models mentioned above. For example, you could ask the learner how they think things went. This will give you insight into their experience and enables you to assess how well they can judge their actions, behaviour or performance. Encourage self-reflection as this will allow the learner to be mindful of their actions or behaviour (table 3). As a rule, start with the positive and then move on to negative events. Be very specific and give examples of certain actions or situations, and use "I" when giving feedback.

"When you said..., I thought that you were..."

Moreover, link the feedback to the learner's overall development and/or stated outcomes, which might provide an additional reason for being receptive to the feedback.

Finally, be aware of nonverbal clues, such as your facial expression, body language, posture, voice and eye contact. These might convey their own message, which could be in contrast with what you are trying to achieve.

- Emotions are deduced through facial expression. Thus, smile in the correct manner to express warmth and goodwill.

- Eye contact creates a feeling of connection but can also be too intense. Make eye contact without staring.

- The way we feel about different people effects the way we speak. The tone and the volume of your voice can give away how you feel. Try to match body language, how you use your voice and tone in a natural way.

- Avoid looking strict by keeping your arms crossed or like you don't care by sitting slumped. An open posture gives the expression of an open conversation.

- Stressed situations make us breathe faster; when we are tired, it is easy to sigh more. Long breaths make us calmer and are likely to make people around us calmer. Try to take a few deep breaths before giving feedback.

- Smartphones and screens with emails, Facebook messages, recent meetings or phone calls: there are so many things to steal our attention and this can only be evident. The person in front of you will notice when you are looking at the screen, and you are less likely to hear and understand what they are saying. Focus your attention on the person in front of you when giving feedback and when you are listening. After giving the feedback, reflect on how it went. Did it go as you expected and how do think it was perceived by the learner? Sometimes, you can misjudge the delivery of your feedback but make sure you learn from this for the next time. Every learner is different and requires a different approach.

In the end, remember to summarise the session in a letter/e-mail and to follow up on what has been discussed. Feedback aims to improve performance and it is advised that time is given to the recipient of feedback to rectify behaviours, then proceed with a follow up session to measure whether or not that is happening, and then make adjustments as you go.

\section{Receiving feedback}

A learner-centred approach is often recommended to effectively receive feedback. This involves adopting an open-minded listening strategy, reflection and a willingness to improve one's performance. The recipients of the feedback are asked to evaluate their own performance and assess how their actions impact others. This approach works best when the feedback is ongoing, regular, supportive, and originating from a wide range of reliable and valid external sources. When this is not the case, the learners may not have sufficient understanding to self-assess and correct behaviours that may hinder their development. However, when constructive feedback is used wisely, it can positively impact the learners' personal and professional development.

It is very helpful to receive feedback from leaders/teachers in real time and firsthand. When the learner is unable to respond positively, however, this often inhibits the feedback providers from giving direct face-to-face, personalised feedback on a regular basis. Learners' responses to criticism may present in negative ways (i.e. anger, denial, blaming or rationalisation), particularly when they discount their own ability to take responsibility for their learning. It is important to view feedback as a means 
Table 4 Tips for receiving feedback

Be a good listener

When in doubt, ask for clarification

Embrace the feedback session as a learning opportunity

Remember to pause and think before responding

Avoid jumping to conclusions, and show that you are invested in the learning process and keen to improve

Think positively and be open to helpful hints

Learn from your mistakes and be motivated

Be a good sport and show appreciation

Be proactive your own actions in the context of the feedback provider's comments is beneficial to making appropriate changes.

\section{Remember to pause and think before responding}

Your aim is to have a professional conversation that benefits you. Focus on understanding the feedback first, not on your immediate innate reaction. Reflection, and particularly self-reflection, is essential to feedback acceptance.

\section{Avoid jumping to conclusions, and show that you are invested in the learning process and keen to improve} on previously learned competencies. The result wil be increased confidence and independence, while facilitating a stronger rapport with colleagues, other medical staff and patients.

In order for feedback to be effective, it has to be received well. How a recipient interprets and reacts to feedback is very important to the outcome of the teacher-learner relationship and future learning opportunities. Differing interpretations or uptakes of feedback may be based on a number of factors that include: personality, fear, confidence, context and individual reasoning processes. It is essential to develop an open dialogue between the person giving feedback and the recipient. Differences of opinion should be handled in a professional manner. Both parties should be comfortable, and able to focus on actively listening, engaging, reflecting and developing action points for future development. Effective communication is key to a successful feedback interaction.

Table 4 presents tips to the learner on receiving constructive feedback.

\section{Be a good listener}

First, truly listen to what the feedback provider is saying, instead of immediately preparing a response, defence or attack. The feedback provider will feel more comfortable giving feedback if you are approachable and welcoming.

\section{When in doubt, ask for clarification}

If you did not hear it clearly the first time, politely ask for it to be repeated, then restate it in your own words. This will help you understand more about yourself and how others interpret your actions.

\section{Embrace the feedback session as a learning opportunity}

Assume that the feedback is constructive until proven otherwise, then consider and use those elements that are truly constructive. Thinking about
Ask for clarification and examples if statements are general, unclear or unsupported. It is important to validate the feedback by inviting details and specifics about the criticism. Assuming the feedback provider's comments as reality, in the context of their perceptions and impressions, defuses your own negative feelings in the face of criticism.

\section{Think positively and be open to helpful hints}

You will get more out of the feedback session if you accept the comments positively (for consideration) rather than dismissively (for self-protection). You may disagree with the criticism if the facts are incorrect but this should be done in a graceful manner.

\section{Learn from your mistakes and be motivated}

Ask for suggestions of ways you might modify or change your behaviour. Do not be afraid to ask for advice on what and how to do differently. Seek to meet expectations and promptly address the undesired behaviours.

\section{Be a good sport and show appreciation}

Be respectful throughout the discussion and thank the person giving feedback. Being polite and appreciative will encourage future feedback.

\section{Be proactive}

Try out some of the suggestions, and make careful notes regarding any improvements and changes in behaviour. Following-up with the feedback provider enables the receiver to share how the feedback was helpful. 


\section{Conclusion}

Giving and receiving effective feedback are skills that are central in healthcare settings. The whole process is closely linked with professional development and improved performance. Both of these impact the quality of healthcare services and patient satisfaction. Feedback should be constructive by focusing on behaviours that can be improved. Developing robust professional relationships is a prerequisite for giving/receiving constructive feedback that will act as a powerful motivator.

\section{Conflict of interest}

None declared.

\section{Suggested reading}

1. Seppala E. When giving critical feedback, focus on your nonverbal cues. https://hbr.org/2017/01/when-givingcritical-feedback-focus-on-your-nonverbal-cues Date last updated: January 20, 2017.

2. University of Sheffield. Giving feedback - some practical tips. www. sheffield.ac.uk/polopoly_fs/1.79368!/file/Giving-Feedback.pdf

3. Healthfield SM. Received feedback with grace and dignity. www.thebalance.com/receive-feedback-with-grace-anddignity-1916643 Date last updated: October 17, 2016.
4. Cornell University Faculty and StaffAssistance Program. How to receive critical feedback. https://fsap.cornell.edu/resources/ upload/How-to-Receive-Critical-Feedback.pdf

5. Dartmouth College. Tips for giving and receiving feedback effectively. www.dartmouth.edu/ hrs/profldev/performance management/feedback.pdf

6. Sargeant JM, Mann KV, van der Vleuten CP, et al. Reflection: a link between receiving and using assessment feedback. Adv in Health Sci Educ 2009; 14: 399. 